\title{
Novel Coordinated Voltage Control for Hybrid Micro-Grid with Islanding Capability
}

\author{
Khaled A. Alobeidli, Student Member, IEEE, Mazheruddin H. Syed, Student Member, IEEE, Mohamed \\ Shawky El Moursi, Member, IEEE, and Hatem. H. Zeineldin, Member, IEEE
}

\begin{abstract}
This paper proposes a new Coordinated Voltage Control (CVC) method with Reactive Power Management Scheme (RPMS) for a Hybrid Micro-grid (MG). The CVC scheme, based on synchronizing the response speeds of different voltage regulating devices, is coordinated with a novel Reactive Power Management Scheme (RPMS). Two cases, with and without proposed CVC, were simulated in the PSCAD/EMTDC environment and compared against each other. The case with proposed CVC shows superior performance, when tested for fault triggered islanding, intentional islanding and MG internal fault. Further, the proposed CVC with RPMS is compared to a voltage regulation method present in literature. The proposed $\mathrm{CVC}$ with RPMS provides better voltage regulation, maximizes the fast dynamic reactive power reserve, and improves the transient response and transient stability margin of the Hybrid Micro-Grid.
\end{abstract}

Index Terms - Coordinated Voltage Control, Distributed Energy Resources, Hybrid Micro-Grid and Grid Codes.

\section{INTRODUCTION}

$\mathrm{T}$ HE continuous integration of low carbon energy resources in the present day power system, although promises to meet the global targets set by countries to reduce their carbon emissions, poses a significant challenge for the Distribution Network Operators (DNO) to maintain satisfactory operation of the network [1]. The combination of widely dispersed low carbon energy resources with large concentrations of modern/future loads, such as heat pumps and electric vehicles, results in a network more susceptible to voltage sags and swells [2]. These voltage sags and swells violate the upper and lower voltage limits set for nominal operation of the network.

The sags and swells in the voltage are mitigated, at distribution level, by means of local voltage controlling devices, such as On Load Tap Changers (OLTC) at the substation [3]. Methods to coordinate the operation of OLTC with SVC, STATCOM and capacitor (CAP) bank to enhance the voltage regulation have been proposed in [4-6]. Maintaining the voltage of the network within acceptable limits by means of controlling the reactive power of Distributed Energy Resources

K. A. Alobeidli, M. S. El Moursi and H. H. Zeineldin are with the Department of Electrical Engineering and Computer Science, Masdar Institute of Science and Technology, Abu Dhabi, UAE, PO Box 54224 (e-mail: melmoursi@masdar.ac.ae). H. H. Zeineldin is currently on leave from the Faculty of Engineering, Cairo University, Giza, Egypt.

M. H. Syed is with the Institute of Energy and Environment, Electrical and Electronics Engineering Department, University of Strathclyde, Glasgow, G1 1XQ, UK (e-mail: mazheruddin.syed@strath.ac.uk ).
(DER) has been presented in [7] and [8]. In [9], the coordinated control of substation OLTC and reactive power of DER to regulate the voltage is proposed. In [10-12], both active and reactive powers of DER are coordinated with the OLTC to achieve robust voltage regulation. With the increase in number of devices capable of providing voltage support, coordinating these devices becomes a complex task. In literature, several optimization techniques (such as linear programming, non-linear programming, genetic algorithm and particle swarm optimization) with different objective functions have been proposed as the solution [13]-[25].

In a Micro-Grid (MG) islanded mode of operation, the problem of voltage is more complex as the $\mathrm{X} / \mathrm{R}$ ratio of the system is low; hence voltage is not only coupled with reactive power, but also with active power and MG frequency [26]. The provision of stable voltage nodes within minimum and maximum voltage limits is important [27]. The stability margin at the system voltage node is decided by the availability of reactive power. According to the $\mathrm{P}-\mathrm{V}$ curves, a power system might be at a critical point depending on the load power factor, where any further decrease in voltage could lead to the operation in a voltage instable region [28]. Hence, in presence of voltage controlling devices, the stability depends on the capability of providing reactive power to the system at the instant required. Therefore, as voltage is required to be stable all the time, reactive power must not be provided only during steady state (SS) condition but also during load changing conditions and faults to avoid voltage instability and/or collapse at any instant [29],[30]. The previous work in literature is more focused on a longer term (steady state) voltage regulation of the distribution network. They did not take into consideration the potential role of slow response compensation devices (OLTC and CAP banks) in providing dynamic support to the network in coordination with fast response devices without deploying communication infrastructure.

This paper presents a new Coordinated Voltage Control (CVC) method with Reactive Power Management Scheme (RPMS), which, by synchronizing the different voltage regulation devices response times, achieves enhanced voltage profile and transient response without deploying a communication infrastructure. The performance of the proposed CVC with RPMS is evaluated for steady state, fault triggered islanding, and intentional islanding followed by an internal fault. Further, the performance of the CVC with RPMS in adhering to the grid codes has also been analyzed. 


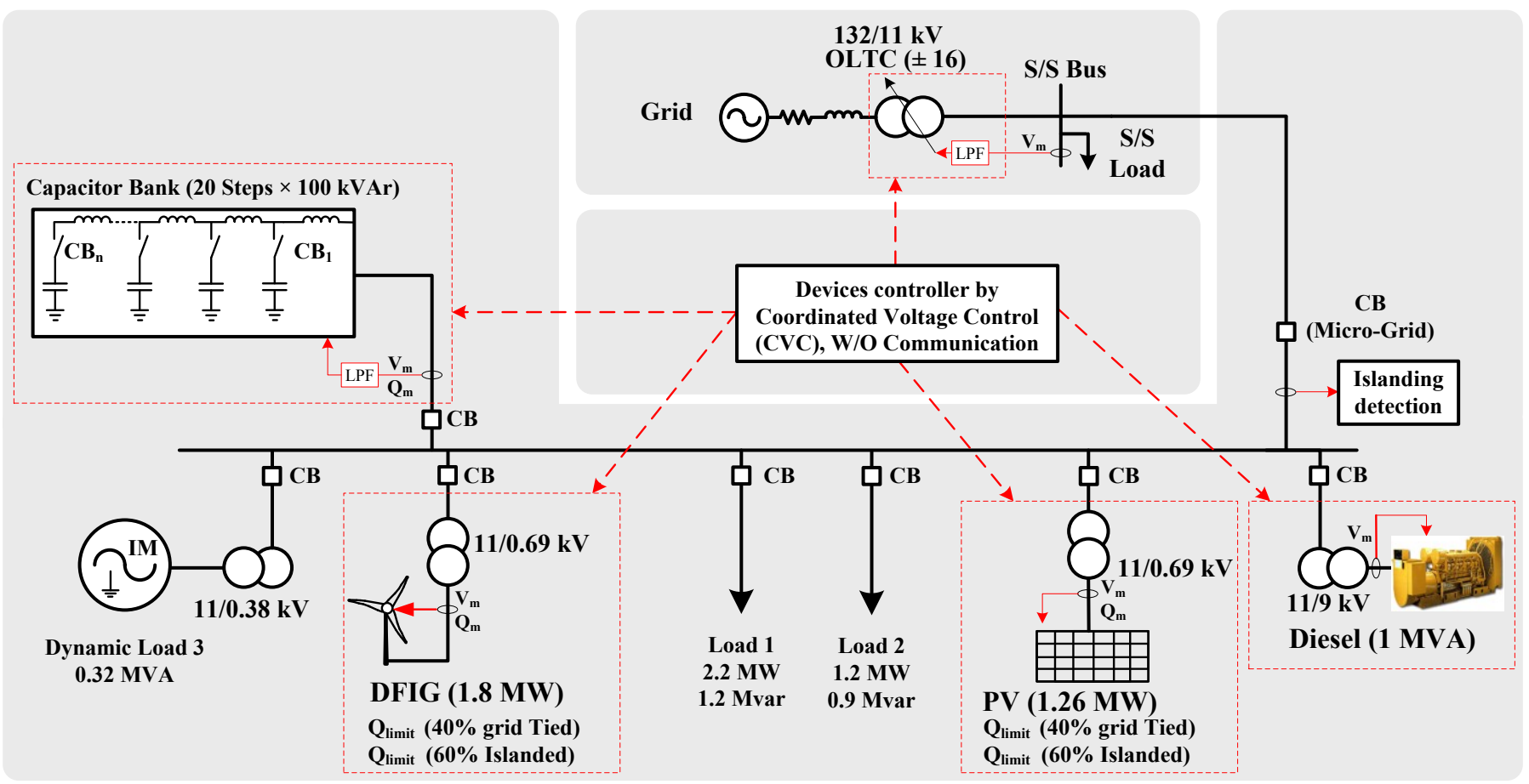

Fig.1. Hybrid MG connected to power system grid.

A comprehensive simulation study is presented that demonstrates the effectiveness of the proposed CVC with RPMS for improving the voltage profile, adhering with the grid codes, maximizing the dynamic reactive power reserve, enhancing the transient response and transient stability margin. The remainder of the paper is organized as follows. The power system and hybrid MG have been described in section Error! Reference source not found.. An overview of the grid codes has been presented in section III. In section IV, the proposed CVC with RPMS is explained in detail. In section V, the implemented islanding detection scheme is presented. The simulation results and evaluation of CVC with inbuilt RPMS is presented in Section VI. Section VII concludes the paper.

\section{II.MICRO-GRID DESCRIPTION}

The layout of the hybrid Micro-Grid under study is shown in Error! Reference source not found.. The utility grid is an equivalent grid of Short Circuit Ratio $(\mathrm{SCR})=14$ and $\mathrm{X} / \mathrm{R}$ ratio $=7$ connected to the radial feeder through a step down transformer $(132 / 11 \mathrm{kV})$ equipped with \pm 16 taps (OLTC). The substation loads are represented by an aggregated load at the substation bus. The hybrid MG is connected to the utility grid by means of a circuit breaker as shown in Error! Reference source not found. The hybrid MG comprises of three different types of generation units: DFIG rated at 2 MVA, PV plant rated at $1.26 \mathrm{MW}$ and $1 \mathrm{MVA}$ Diesel Generator with implicit transformer. The inverter-based DER units (PV and DFIG) generate power through $0.69 / 11 \mathrm{kV}$ step up transformers, while the Diesel generator and the dynamic load are connected through a $9 / 11 \mathrm{kV}$ and a $0.38 / 11 \mathrm{kV}$ step up transformers respectively. The loads in the MG comprise of a mix of fixed (L1 and L2) and dynamic loads (L3-Induction Motor).

The OLTC model is based on a discrete controller such that if the measured voltage is out of a specified dead-band, the voltage will be regulated based on the stepwise tap movements and delay mechanism as explained in [31], [32]. Mechanically switched capacitor bank with 20 steps of $100 \mathrm{kVAr}$ each (i.e. 2 MVAr) represents the CAP bank. This model is equipped with current reactors to limit the currents during switching in (inrush current) and fault (outrush current). Each step is switched at almost zero voltage (Zero Voltage Switching). A similar discrete controller to OLTC, with specified dead-band and time delay, controls the switching of the 20 capacitor stages in CAP bank. The Diesel generator model consists of a speed governor, excitation system and a synchronous machine. The desired mechanical torque is regulated by controlling the fuel intake. Woodward governor model has been employed for this work. The excitation system is used to regulate the system voltage at the desired level. The inverter-based DER units (PV and DFIG) have been modeled as an average model using a Controllable Voltage Source (CVS) with DC link dynamic circuit. The inverter-based DER (PV) employs decoupled current control. It is equipped with an outer voltage control loop and an inner current control loop in the dq reference frame, where the direct component of the current controls the dc link voltage and the quadrature component of the current controls the desired measured voltage of the system. It is worthy to mention that during islanded operation the Diesel generator is dedicated to frequency regulation in the MG.

\section{GRID CODES}

Grid codes are set by Distribution Network Operators (DNO's) or Transmission System Operators (TSO's) in order to maintain proper functionality of the grid. Grid codes set out certain technical and organizational requirements to be fulfilled 
by a generating unit operator willing to connect and operate parallel to the DNO's or TSO's grid. Grid codes serve as a basis for planning and operation of generating units at the grid. This section describes the requirements of a generating plant to operate at the medium voltage network set out by German Association of Energy and Water Industries (BDEW) in 2008 [32]. In particular, the dynamic network support and protection requirements have been presented in detail.

Medium voltage network refers to a network with operating voltages in the range of $10-30 \mathrm{kV}$. These grid codes are applicable to all generating units connected to the medium voltage network directly or indirectly. If a plant is generating at a lower voltage but is connected to the medium voltage network through a step up transformer, it has to abide by the medium voltage grid codes. A generating plant may be a single generating unit or a group of generating units, may comprise of synchronous or asynchronous generators and/or inverter-based DERs. There is no generic minimum or maximum power generation capacity requirement to operate at the medium voltage network. It depends on the type, control and location of the generating plant and is upon the discretion of the grid.

Generating plants have been distinguished into two by the grid codes, namely, Type 1 and Type 2. A synchronous generator connected directly to the network is considered to be Type 1. All other systems (for example, a hybrid MG with synchronous generator, inverter-based DER units) fall under Type 2. As the main focus of this paper is on hybrid Micro-Grids, the remaining of this section will be in relevance to Type 2 installations.

\section{A. Dynamic Network Support}

A generating plant connected to the medium voltage network should be able to provide dynamic support to the network in case of disturbances in the network. Dynamic network support refers to the following:

- A generating plant should stay connected to the grid during a fault (all types of faults).

- A generating plant should support the voltage by injecting reactive power.

- A generating plant, post clearance of a fault, should consume same or less reactive power as the pre-fault condition.

For a fault duration of $\leq 150 \mathrm{~ms}$ with zero voltage, a generating plant can neither disconnect itself from the grid nor cause instability. A generating plant needs to stay connected during the fault and provide reactive power support to the network. In order to understand the dynamic network requirements clearly, let us consider the limiting curves of a generating plant during a fault as shown in

Fig.1. A generating plant with voltage profile (during and subsequent to a fault) above borderline 1 cannot disconnect itself or lead the system to instability. On the other hand, a generating plant with voltage profile (during and subsequent to a fault) between border line 1 and 2, may be allowed to disconnect for a short duration of time, up to 2 seconds if necessary (if disconnection is necessary to prevent damage to either network or to the generating plant).

\section{B. Protection}

For secure and reliable operation of a network and generation units, the protection devices play a crucial role.

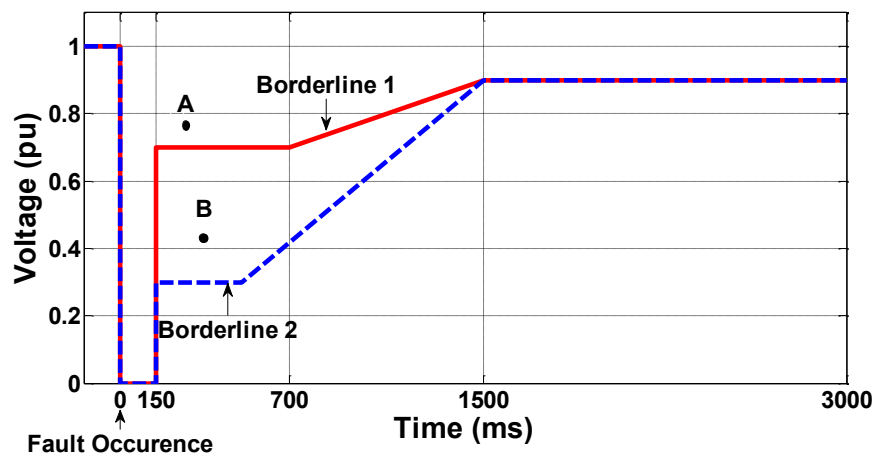

Fig.1. The fault ride through curves of the voltage at PCC for a Micro-Grid operation in response to grid faults

The protection devices are responsible for disconnection of generation unit from the medium voltage network in case of disturbed operating conditions (e.g. network faults), to prevent any damage to the generation unit and other customers of the medium voltage network.

A generation unit operator is responsible for protection of his generation unit. The generation unit operator needs to ensure adequate amount of protection equipment installations as shown in Fig. 2. For generation units capable of islanded operation, the protection units installed need to guarantee proper protection for islanded mode of operation. Thus, Under/over voltage and frequency relays are required to ensure proper operation of the network. The location of the protective devices depends upon several factors, one of them being the possibility to operate as a standalone system. The recommended protection settings provided by the grid codes for protection unit at Point of Common Coupling (PCC) and the generating units are presented in Error! Reference source not found. and

Table II respectively.

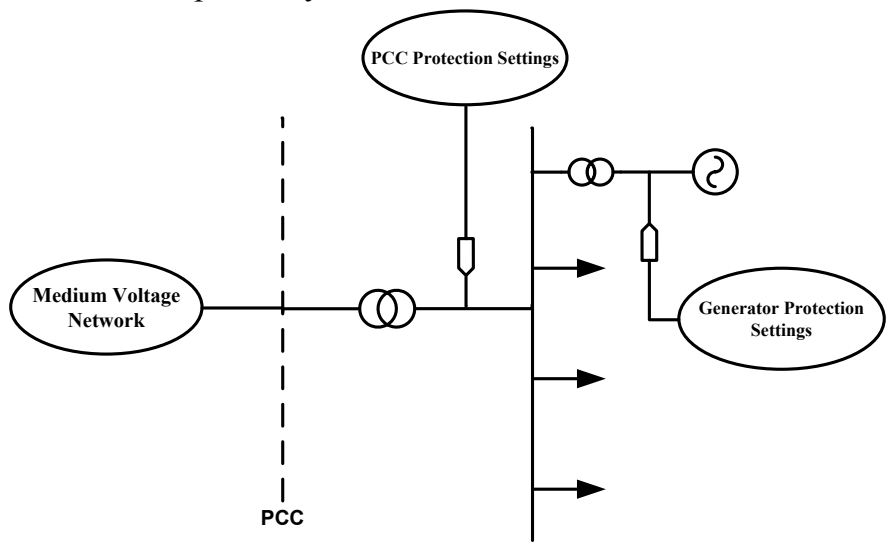

Fig. 2. The schematic diagram of the Micro-Grid connection and PCC protection settings for the deployed islanding detection scheme.

\section{COORDinAted VOlTAge CONTROL}

The objective of CVC is twofold 1) to maximize the utilization of each component in the system 2) to maximize the dynamic reactive power reserve in the system. In order to maximize the 


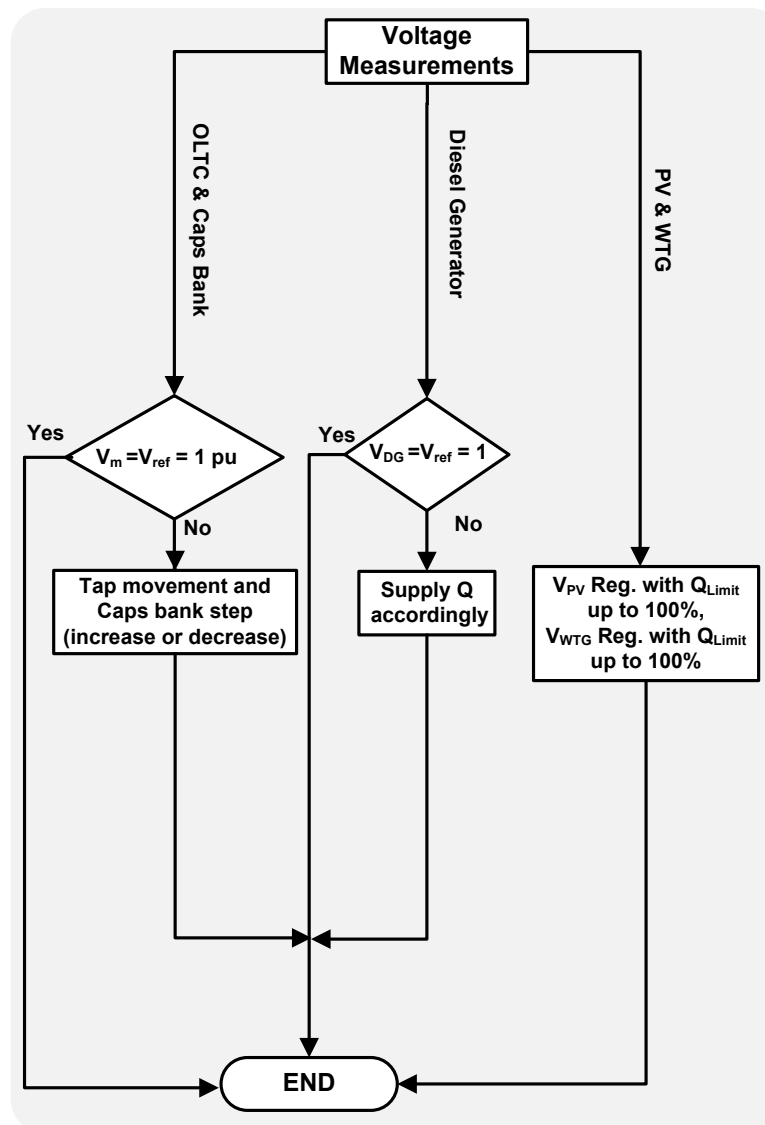

(a)

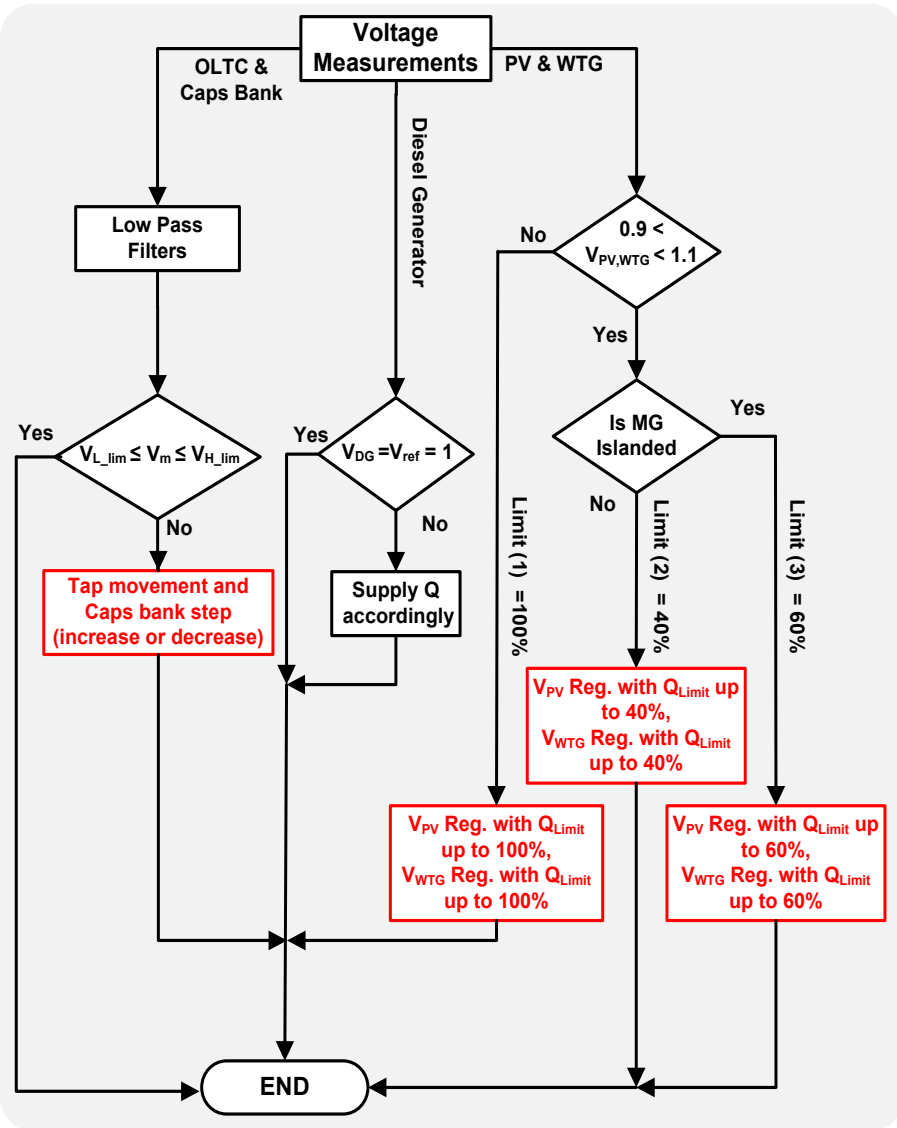

(b)

Fig.4 The proposed control algorithm for a Hybrid MG: a) without CVC and RPMS and b) with CVC and RPMS.

utilization of each component in the MG, it is necessary to identify the states of the system.

The three well known states in power system are steady state, load dynamic state and fault state. Each voltage regulating component in the MG (i.e. CAP bank, OLTC, Diesel generator, PV and DFIG) has a different response time. The OLTC and CAP bank are slow devices. The diesel generator has medium voltage response whereas the PV and DFIG are capable of instantaneous reactive power compensation owing to the inverters capabilities.

The aim here is to relate the three mentioned states of power

Table I

Recommended Protection Settings at PCC

\begin{tabular}{ccc}
\hline \hline Protection Function & \multicolumn{2}{c}{ Recommended Protection } \\
& \multicolumn{2}{c}{ Settings } \\
\hline \hline Rise in Voltage $(V \gg)$ & $1.20 \mathrm{pu}$ & $100 \mathrm{~ms}$ \\
Rise in Voltage $(V>)$ & $1.08 \mathrm{pu}$ & $1 \mathrm{~min}$ \\
Under Voltage $(V<)$ & $0.8 \mathrm{pu}$ & $2.7 \mathrm{~s}$ \\
\hline \hline
\end{tabular}

Table II

Recommended Protection Settings at Generating Unit

\begin{tabular}{ccc}
\hline \hline Protection Function & \multicolumn{2}{c}{ Recommended Protection } \\
& \multicolumn{2}{c}{ Settings } \\
\hline \hline Rise in Voltage $(V \gg)$ & $1.20 \mathrm{pu}$ & $100 \mathrm{~ms}$ \\
Under Voltage $(V<)$ & $0.8 \mathrm{pu}$ & $1.5-2.4 \mathrm{~s}$ \\
Under Voltage $(V \ll)$ & $0.45 \mathrm{pu}$ & $300 \mathrm{~ms}$ \\
Rise in Frequency $(f>)$ & $1.03 \mathrm{pu}$ & $100 \mathrm{~ms}$ \\
Under Frequency $(f<)$ & $0.95 \mathrm{pu}$ & $100 \mathrm{~ms}$ \\
\hline \hline
\end{tabular}

system to the response time they require. During steady state and minor dynamics in the system (e.g. load change), the regulation of voltage can be provided by any of the slow devices in the system. The term steady state, in this paper, refers to the state when the measured voltage $\left(\mathrm{V}_{\mathrm{m}}\right)$ is within $\pm 10 \%$. Therefore, the response time of the voltage regulating device during steady state is not a critical concern; hence for maximum utilization it is desirable to induce the OLTC and CAP bank to regulate the voltage in steady state and the Diesel generator in minor system dynamics within the operating voltage criteria (0.9pu-1.1pu). Consequently, it is not desirable to use fast response devices to regulate the voltage during steady state and minor load switching transients. This is due to the fact that; it can saturate the reactive power compensation capability of the inverter-based DER units and render them ineffective during transients. The stability of the system is a critical concern and is a priority. The response time of OLTC, CAP bank and Diesel generator are slow, compared to inverter-based devices, to support the system with reactive power compensation during a fault that lasts for around $150 \mathrm{~ms}$. It is desirable to have maximum possible fast compensating reactive power reserve for fault transients.

From the above discussion, it is clear that a scheme to coordinate the response of voltage control devices is a necessity for efficient utilization of available resources. Therefore, the CVC scheme presented in this paper aims to coordinate the responses of all voltage control devices in the system to 
maximize each components utilization and overall fast dynamic reactive power reserve. In the following subsections the operation of the system without and with CVC is explained. This is done for the purpose of comparison, to show the effectiveness of the proposed CVC and RPM schemes. The control scheme for both cases is depicted in Error! Reference source not found. $(a, b)$.

\section{A. Case 1: Without CVC (W/O CVC)}

In this case, the voltage controlling devices are not coordinated and they regulate the voltage based on their maximum capabilities. The MG voltage can be controlled by all DER units, OLTC and the CAP bank without taking into consideration the response speed priority of system states: steady state, dynamics and system contingencies. The reactive power support from inverter-based devices is not limited and can provide up to $100 \%$ reactive power injection to the MG.

\section{B. Case 2 (CVC with RPMS)}

During steady state, the Reactive Power Management Scheme (RPMS) is implemented on inverter-based DER units. Their reactive power support is limited by the interface control. Therefore, the slow devices (CAP and OLTC) are induced to regulate the voltage in steady state conditions. In addition, the measured voltage for regulation by the OLTC and CAP bank are delayed using Low Pass Filter (LPF). As a result, during steady state they will take more steps, providing more reactive power for better voltage profile and maximizing the reactive power reserve in DER units. The OLTC and CAP utilization during steady state is high. In a MG contingency situation such as faults, the controllers of inverter-based DER units release the reactive power limits (based on the MG voltage i.e. when the voltage is below $0.9 \mathrm{pu}$ ) to their full capability.

The implementation of CVC on MG is adaptive according to the system conditions. The three states of operation are shown in Fig.3 based on voltage level and reactive power limitation of the inverter-based DER units. For grid connected mode, the $I_{q}$ reference signal from the voltage controllers of inverter-based DER units is hard limited to $40 \%$ of its rating in steady state conditions as shown in Fig. 4. However, the limits are released to $100 \%$ reactive power compensation during system dynamics and transient, that is if $\mathrm{V}_{\mathrm{m}}$ is below $0.9 \mathrm{pu}$. The limit of $40 \%$ reactive power support during grid connected mode is set based on the fact that there is reactive power support from the grid.

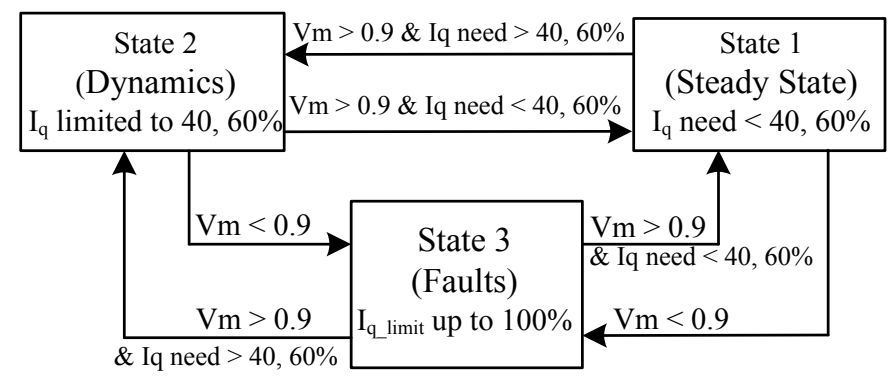

Fig.3. Three states of operation.

As stated earlier, the measured voltage signal to control slow responding devices (i.e. OLTC and CAP bank) are filtered using LPF. In islanded mode of operation, the $40 \%$ reactive power compensation limit during steady state will not be sufficient to regulate the MG voltage, thus it is raised to $60 \%$. Therefore, when the MG breaker opens the circuit, the inverter-based DER units increase the limits to $60 \%$ reactive power during steady state and $100 \%$ during system contingencies. In addition, when the limit is released from $60 \%$ to $100 \%$ the controller will maintain the $100 \%$ limit for 1 second in order to prevent any bouncing effect among generation units.
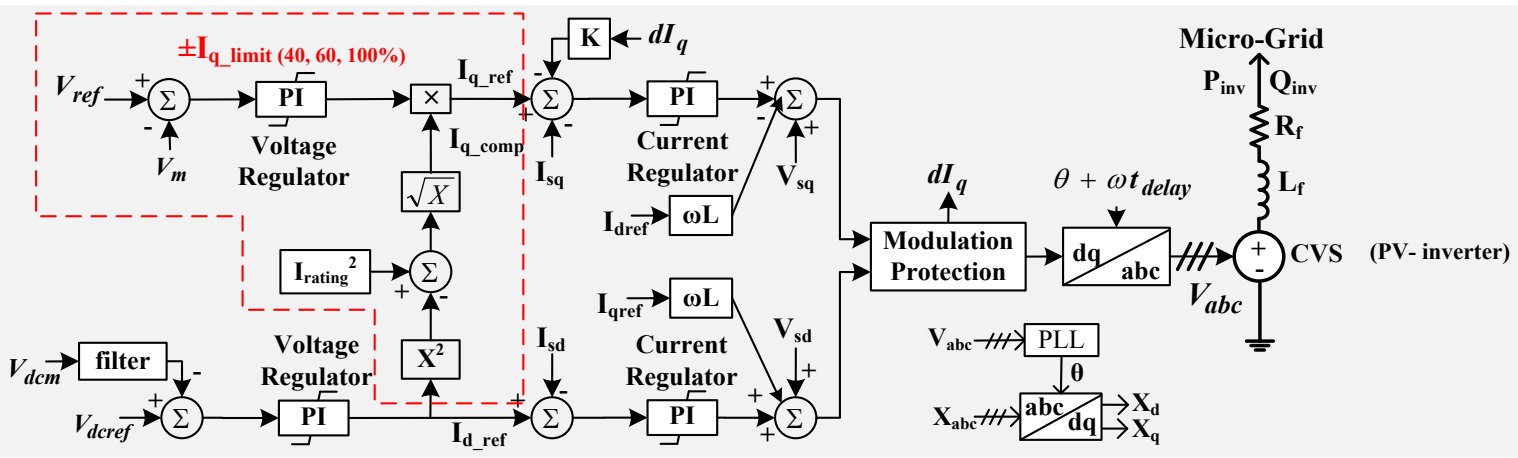

WT - Grid Side Control
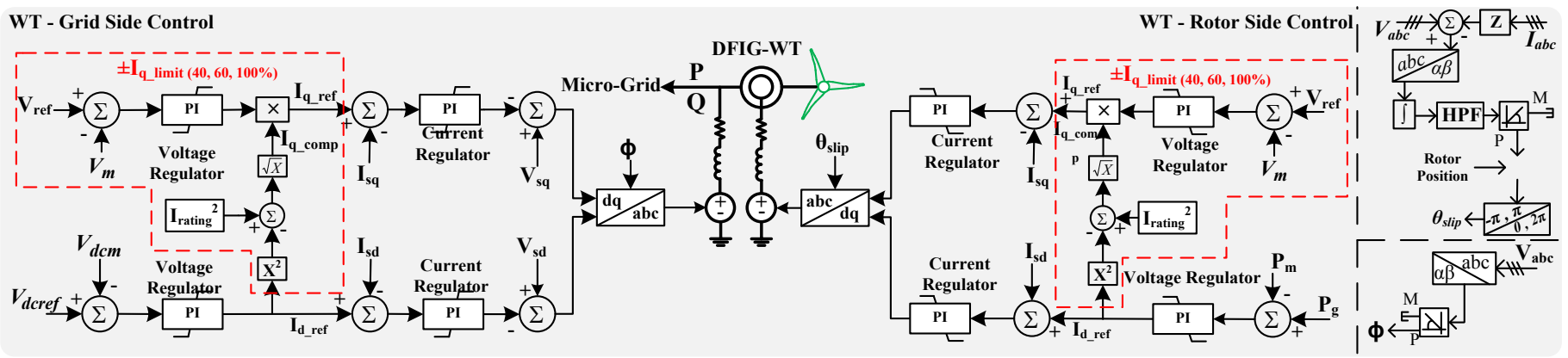

Fig. 4. PV inverter and DFIG controls. 
The steady state reactive power compensation of inverter-based DERs in grid connected and islanded operations of MG have been chosen as $40 \%$ and $60 \%$ respectively. In grid connected operation of the MG, slow (OLTC and CAP banks), medium (Diesel Generator) and fast (PV and DFIG-WT) response regulation devices provide voltage support to the $\mathrm{MG}$. The allowed \% of steady state reactive current compensation from the inverter-based DERs is determined by the following steps:

a. Obtain the voltage profile at MG connection point (PCC) under peak load condition by means of power flow analysis with OLTC, CAP banks, Diesel Generator and inverter-based DERs connected.

b. The inverter-based DERs should be operated at floating power factor with a limit of 0.95 (maximum). While having the OLTC, CAP banks and diesel generator regulating the voltage at $\mathrm{PCC}$, the $\%$ of reactive power compensation from inverter-based DERs is increased until the voltage operating criteria (0.9-1.1 p.u.) is satisfied. This step is carried out considering the following operating conditions:

- Minimum SCR and X/R ratio of the grid.

- The PV inverter and DFIG-WT are operated at maximum active power generation.

○ Voltage control is deployed at rotor and grid side converters of the DFIG-WT.

c. To ensure proper utilization of inductive compensation of inverter-based DERs, power flow analysis is conducted at off-peak load while freezing the OLTC and CAP banks in case of overvoltage. This ensures that the inverter-based DERs are capable to regulate the voltage within permissible operating range and maximize their fast dynamic reactive power reserve by operating in inductive mode of operation.

However, in the islanded operation of $\mathrm{MG}$, system voltage regulation is dependent upon the MG components. There is no support from the grid and the OLTC. Therefore, to obtain the allowed $\%$ of steady state reactive current compensation from the inverter-based DERs, the same afore mentioned steps are followed by excluding the OLTC. The SCR and X/R ratio of the grid is not important as the MG is islanded.

It should be noted that the final \% of steady state reactive power compensation from the inverter-based DERs is rounded to the nearest ten.

\section{V.ISLANDING DETECTION}

One of the major advantages of a Micro-Grid is its ability to operate in islanded mode. A Micro-Grid islanding can be broadly classified as intentional and unintentional islanding. Intentional islanding may take place for maintenance of a grid. Unintentional islanding takes place due to unforeseen disturbances in the network. Detection of islanding has been a challenge for reliable operation of a Micro-Grid. Islanding detection techniques can be distinguished into three: active, passive and communication based. Islanding detection schemes that solely depend on the local measurements at the PCC are referred to as passive islanding detection techniques. On the other hand, active islanding techniques are those that introduce deliberate perturbations in the system to detect islanding. Communication based techniques rely on transmitting and receiving an islanding detection signal to detect islanding. Several Islanding detection schemes have been investigated in literature. Communication based islanding techniques have been investigated in [34]-[37] passive islanding techniques in [38]-[43] and active islanding techniques in [44]-[50].

In this paper a simple passive islanding detection scheme has been implemented which relies on voltage. The scheme has to abide by grid codes as explained in section Error! Reference source not found. The $\mathrm{MG}$ breaker cannot operate to disconnect the MG from the power system for fault duration up to $150 \mathrm{~ms}$. If the duration of the fault exceeds $150 \mathrm{~ms}$, the MG is allowed to disconnect from the power system. Both the inverter-based DER's (DFIG and PV) are equipped with islanding detection. As the islanding detection is based on local voltage measurement there is no need of communication for islanding.

\section{Simulation Results And Evaluation}

In this section the effectiveness of the proposed CVC scheme has been presented and compared to the case without CVC in subsections A, B and C. The CVC is evaluated for load change; fault triggered islanding, intentional islanding and an internal fault (after islanding). Further, the proposed CVC is compared with a voltage regulation method present in literature in subsection D.

\section{A. Load change and fault triggered islanding}

In this subsection, the performance of the system without and with CVC for steady state is presented considering both loads (L2 and L3). Fig. 7 presents the operation of the OLTC with and without the CVC scheme prior to an islanding event. As can be seen, without CVC, the OLTC experience less tap changes and this is due to the contribution from inverter-based DERs. By limiting the reactive power support of these devices (as per the proposed CVC), an increase in tap changes is observed resulting in increasing the fast dynamic reactive power reserve.

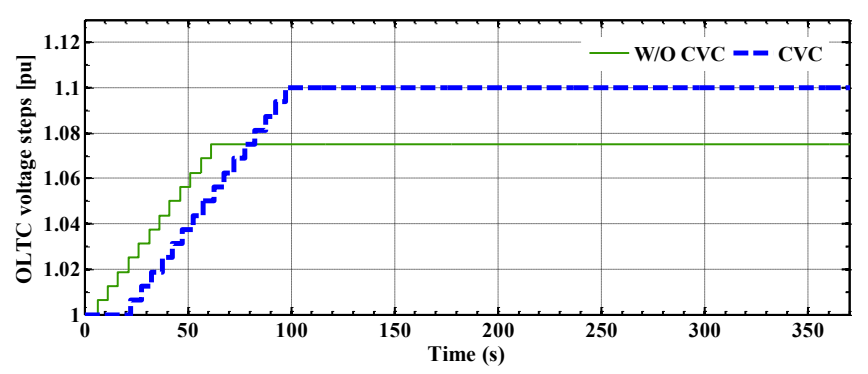

Fig.7. OLTC tap changes during grid connected operation

Fig. 8 shows the voltage at the PCC during steady state and a fault triggered islanding event occurring at $350 \mathrm{sec}$. The reactive current limit (40\%) was deployed on the inverter-based devices during grid connected mode up to $350 \mathrm{sec}$ and changed 
to $(100 \%)$ once a fault triggered islanding event is detected. As seen in Fig. 9 (a), the limitation on reactive power compensation from fast devices in CVC case (during grid connected mode), forced the slower compensation devices (i.e. OLTC \& CAP) to take more steps of taps and capacitor banks to regulate the voltage. The local voltage measurements of the OLTC \& CAP are filtered using LPFs to postpone their response of regulation to enable the fast regulation of the inverter-based DERs within the specified limit.

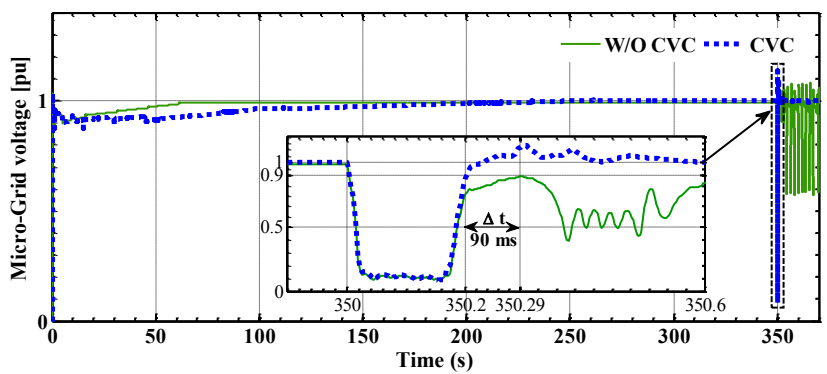

Fig.8. Voltage profile in steady state and fault triggered islanding.

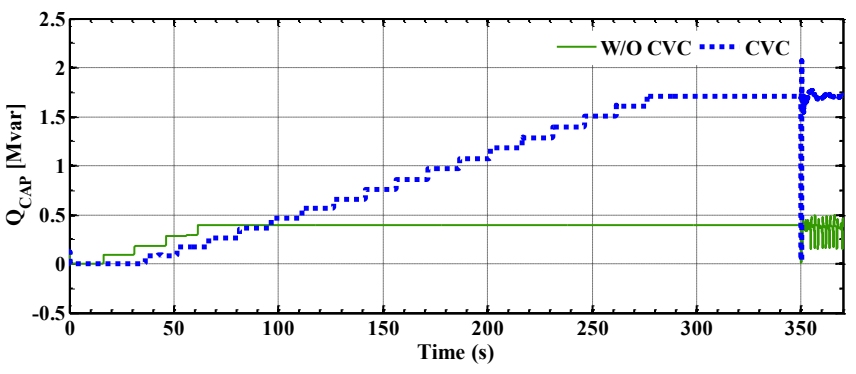

(a)

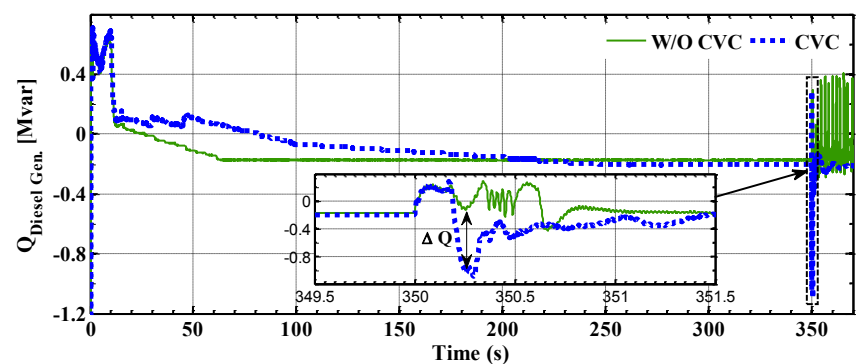

(b)

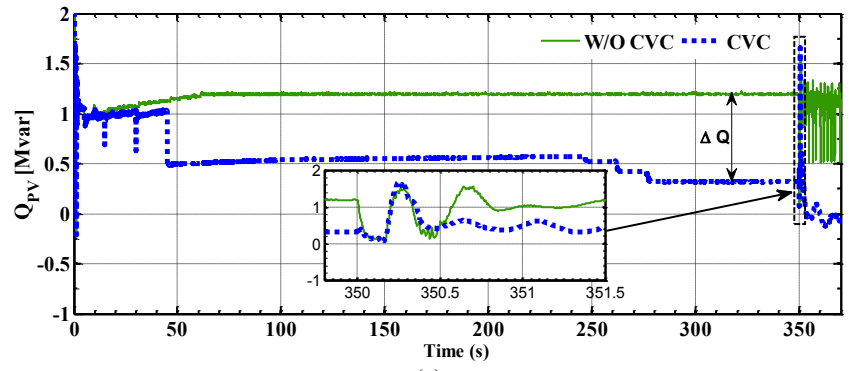

(c)

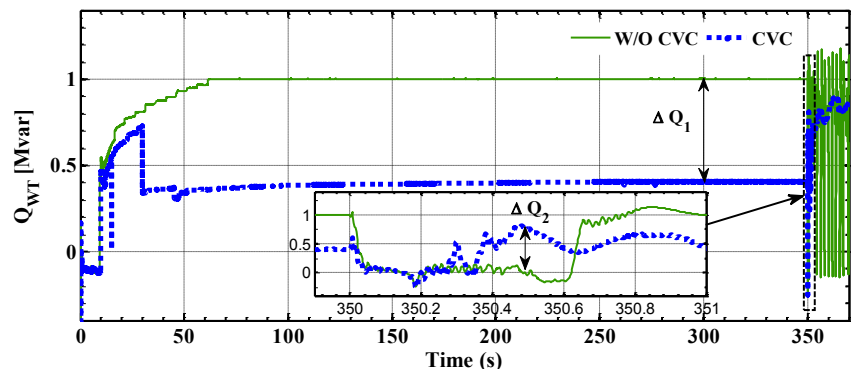

(d)

Fig. 9. (a-d) Reactive power compensation from: (a) Capacitor banks; (b) diesel generator, (c) PV, and (d) WTG.

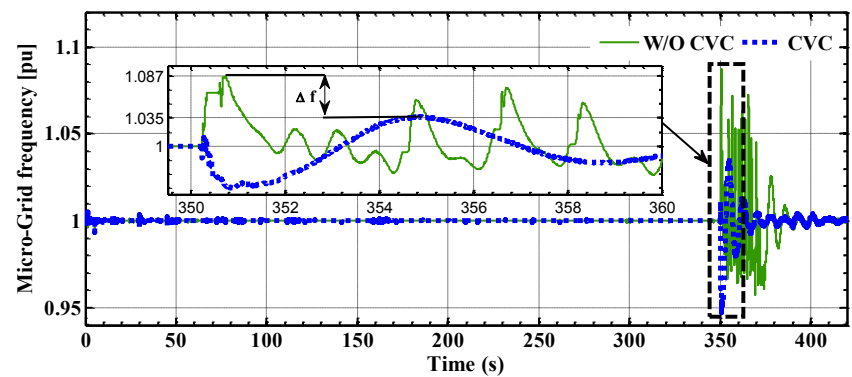

Fig.10. Frequency of the MG in SS and fault triggered islanding.

Thus, it imposed even further regulation to the voltage, which indirectly increases the dynamic reactive power reserve for inverter-based devices. The regulation from OLTC and CAP bank difference (i.e. $\Delta Q$ ) and the reactive power reserve difference (i.e. $\Delta Q_{1}$ and $\Delta Q_{2}$ ) in DER units are shown in Fig. 9 (a-d). The simulation results demonstrate the effectiveness of the proposed control strategy for maximizing the fast dynamic reactive power reserve by $60 \%$ and $75 \%$ in the DFIG-WT and $\mathrm{PV}$ respectively. Also, it reduced the required compensation from the diesel generator resulted in improving the system stability in response to severe system disturbances.

From the voltage recovery profile, it is clearly observed that CVC outperforms the case W/O CVC. The system with employing the CVC ensures faster transient response and system stability unlike the case without $\mathrm{CVC}$ where the $\mathrm{MG}$ tends to become unstable. Also, it helps indirectly to suppress the frequency excursion within the acceptable limit for islanded mode operation as shown in Fig. 10.

\section{B. Intentional Islanding and Internal fault}

To demonstrate the effectiveness of the proposed CVC, it is further tested for intentional islanding and an internal three phase to ground fault for $150 \mathrm{~ms}$. The performance of the CVC is superior compared to the case $\mathrm{W} / \mathrm{O} \mathrm{CVC}$ as shown in Fig.11 with better transient response during intentional islanding. At $400 \mathrm{sec}$, an internal fault is applied and the performance of the CVC is evaluated. The proposed control strategy and reactive power management scheme demonstrate superior performance for enhancing the voltage recovery to 0.9 pu by $290 \mathrm{~ms}$ compared with the case without CVC as shown in Fig.11. The fast dynamic reactive power reserve in the inverter-based DERs helps to improve the transient response as well as the voltage stability of the MG islanded mode of operation. The reactive power from CAP bank, Diesel Generator, PV and DFIG are shown in Fig.12 (a-d). The 
reactive power response of the Diesel generator in Fig.12 (b) shows the difference in the reactive power management of the two cases, where $\mathrm{W} / \mathrm{O} \mathrm{CVC}$ injects more reactive power. However, with $\mathrm{CVC}$ it injects less reactive power and therefore higher dynamic reactive power reserve. The frequency of the MG during intentional islanding shows a sustained oscillation in the case $\mathrm{W} / \mathrm{O} \mathrm{CVC}$ for $3 \mathrm{~s}$, while better damping is illustrated by the CVC performance as in Fig.13. The higher reactive power reserve in the case with $\mathrm{CVC}$ indicated as $(\Delta Q)$ in Fig.12 has resulted in better dynamic and stability performance of the Micro-Grid by improved transient voltage response.

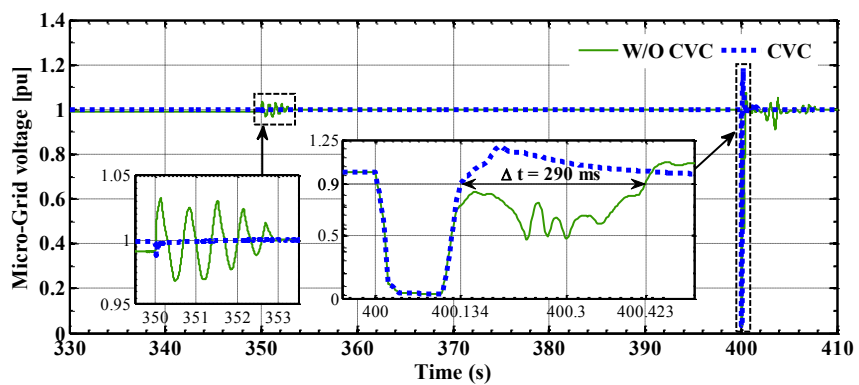

Fig.11. MG voltage in response to intentional islanding and MG internal fault.

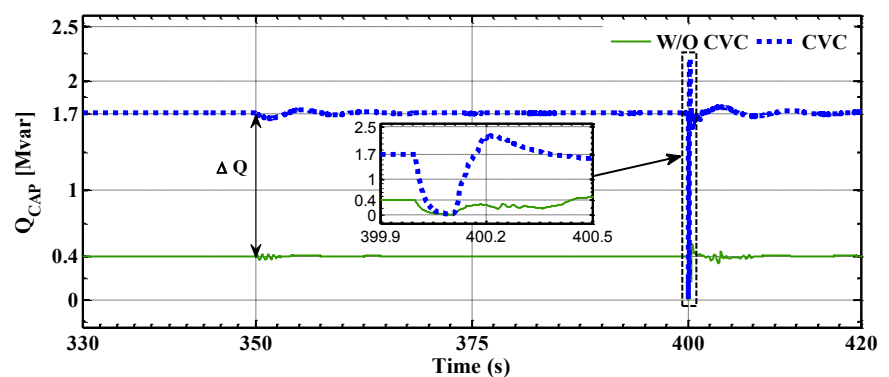

(a)

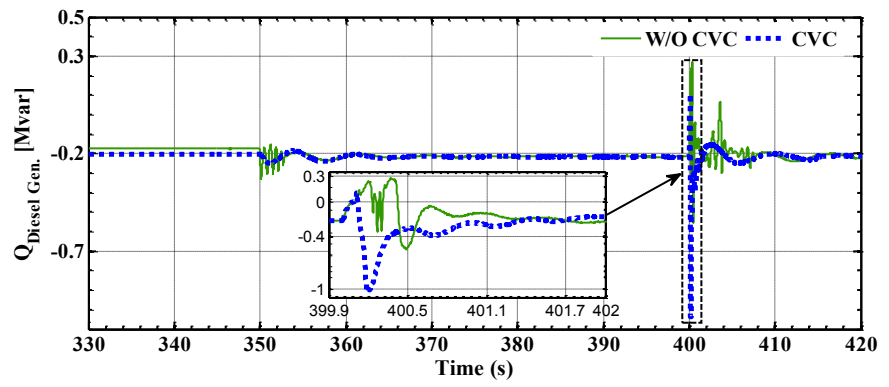

(b)

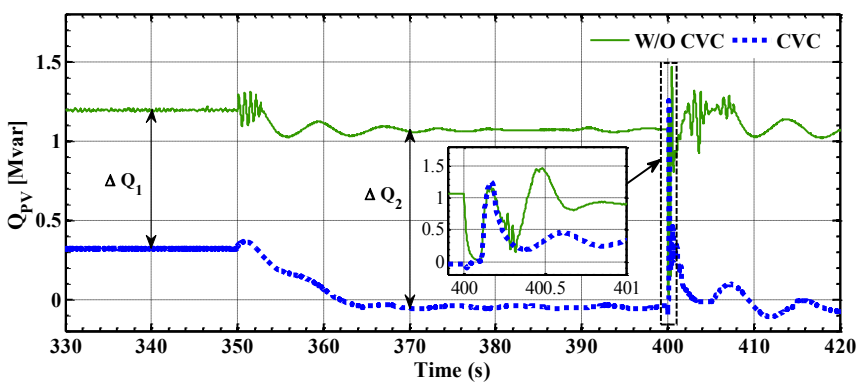

(c)

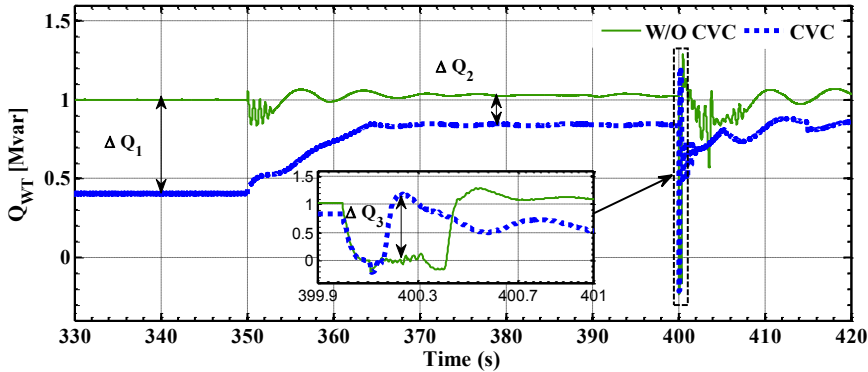

(d)

Fig. 12 (a-d) Reactive power compensation from: (a) Capacitor banks; (b) diesel generator, (c) PV, and (d) DFIG-WTG.

\section{Improving the Transient Stability Margin}

The Maximum Critical Clearing Time (MCCT) is considered as an indicator of the transient stability margin of the MG. In this context, the MCCT of the MG is determined in response to fault triggered islanding as well as internal fault during islanded mode of operation with and without employing the CVC.

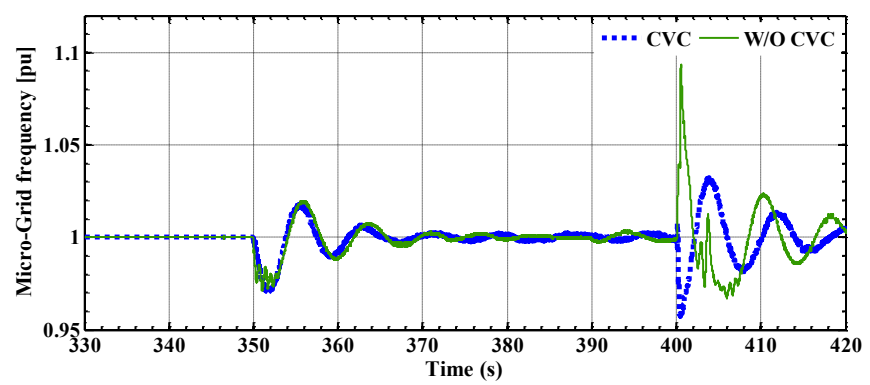

Fig.13. MG frequency in response to intentional islanding and internal fault.

The proposed CVC demonstrates superior performance for improving the MCCT for fault triggered islanding up to $230 \mathrm{~ms}$ and $360 \mathrm{~ms}$ during internal fault under islanded mode of operation as shown in Fig. 14(a-d). The diesel generator speeds are shown for the simulated cases at the MCCT with and without CVC to demonstrate the advantages of deploying such control strategy and reactive power management scheme for MG operation.

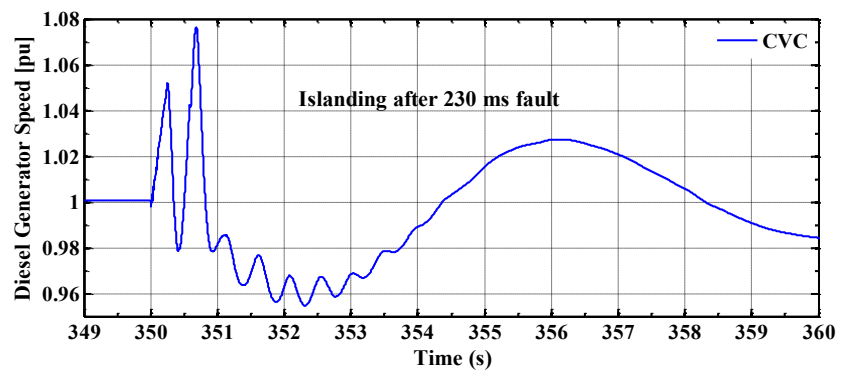

(a) 


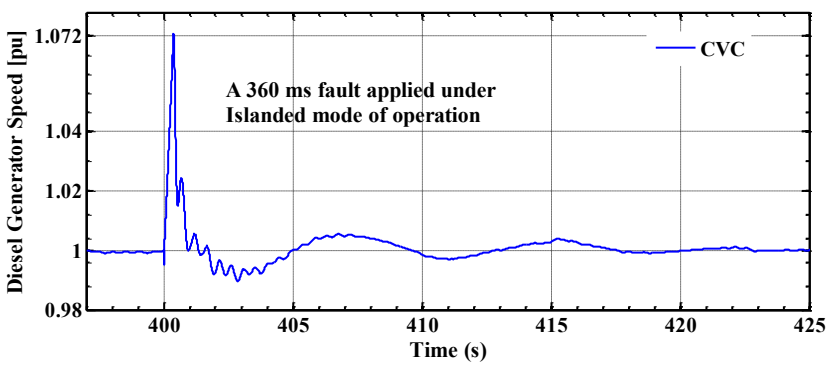

(b)

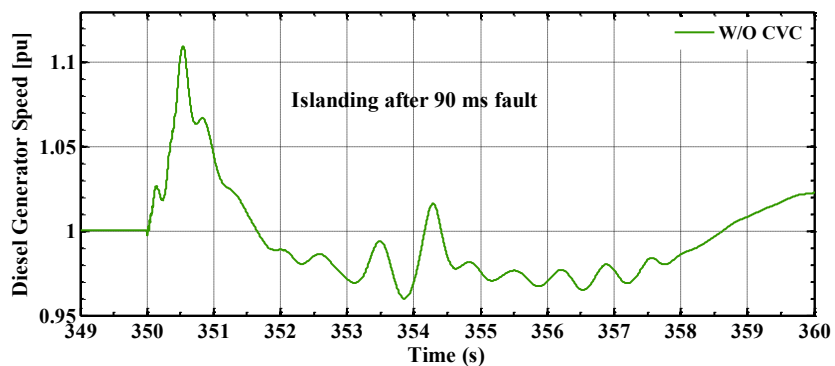

(c)

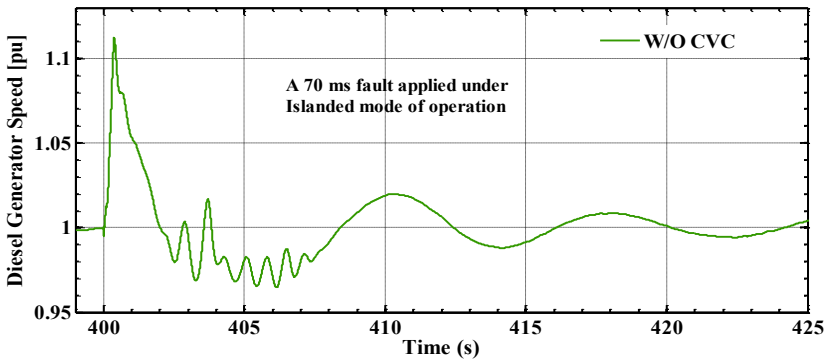

(d)

Fig. 14. (a-d) Diesel generator speed for fault triggered Islanding and fault in islanded mode of operation (a, b) W CVC (c, d) W/O CVC.

D. Advantages of the proposed control schemes over the deployed PF control strategy for a Hybrid MG

The performance of the proposed control strategies of Case 2 is verified with respect to the published method $[11,12]$. Thus, the control strategy of Case 3 is developed as presented in [11, 12]. The rule based method for DERs, OLTC and CAP bank is developed to achieve the desired voltage regulation within a MG as explained in [11]. This method relies on the following control functions:

1. Regulating the MG voltage to the permissible operating ranges using the OLTC and CAP Banks without reactive power support from inverter-based DERs (operated at unity power factor).

2. If the voltage regulation is not satisfactory the inverter-based DERs are used to restore the voltage with the desired limit by releasing the pf up to 0.98 or 0.95 as presented in [11] and [12] respectively. Hence, the inverter-based DERs provide the required reactive power compensation to the MG.

3. The transition from unity pf to 0.98 or $0.95 \mathrm{pf}$ the required reactive power compensation should be calculated based on the state estimation method [11] or applying slope for achieving acceptable transient response especially for islanded operation of $\mathrm{MG}$.

The simulation studies are carried out to evaluate the performance of the reported two cases in response to fault triggered islanding for improving the transient response with alternatively employing the pf control up to 0.98 or 0.95 for Case 3. In the case of change to $0.95 \mathrm{pf}$ instant change creates instability to the weak MG, thus it is made to follow a slope.

Fig. 15 (a, b) demonstrates the superior performance of Case 2 for improving the transient response. It can be observed that the proposed CVC \& RPMS achieves faster voltage recovery of $150 \mathrm{~ms}$ and $270 \mathrm{~ms}$ compared with Case 3 that employed pf control to 0.98 and 0.95 respectively to ensure the desired voltage profile of MG. Fig. $16(\mathrm{a}, \mathrm{b})$ shows the transition of $\mathrm{pf}$ control from unity to 0.98 instantly and to $0.95 \mathrm{pf}$ following a slope of $5 \mathrm{sec}$ for PV and DFIG-WT when the MG triggered islanding based on fault.

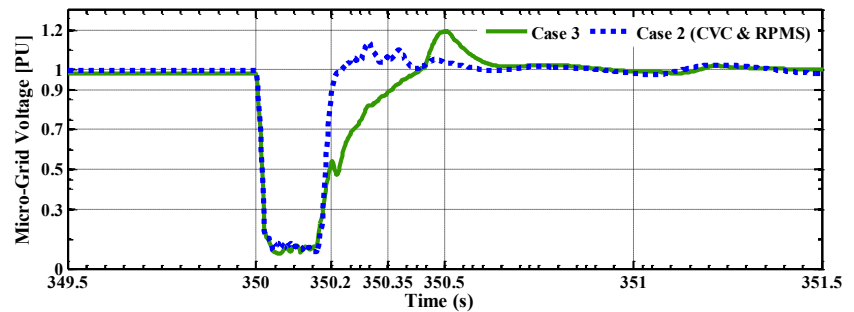

(a)

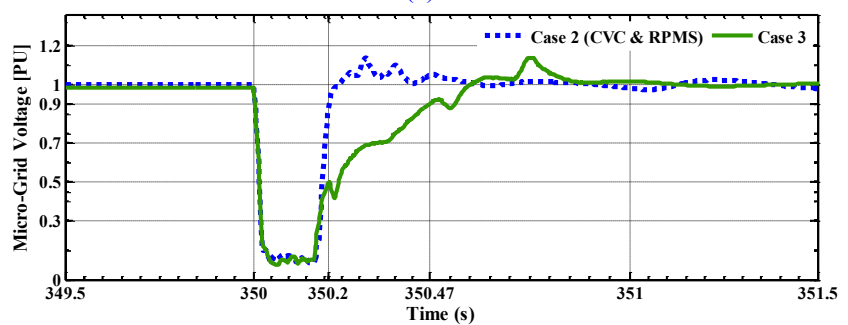

(b)

Fig. 15 The voltage response without and with CVC and RPMS: a) pf control up to 0.98 and b) a) pf control up to 0.95 .

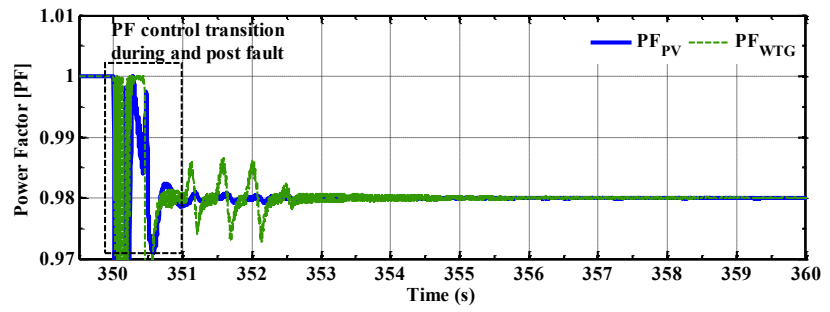

(a)

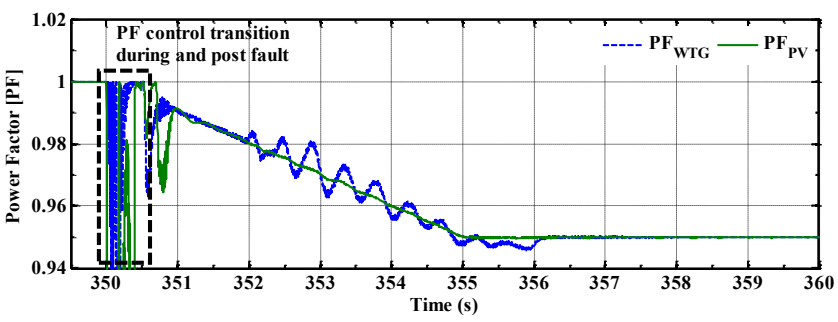

(b)

Fig. 16 The pf control is released for PV and WTG up to 0.98 . 


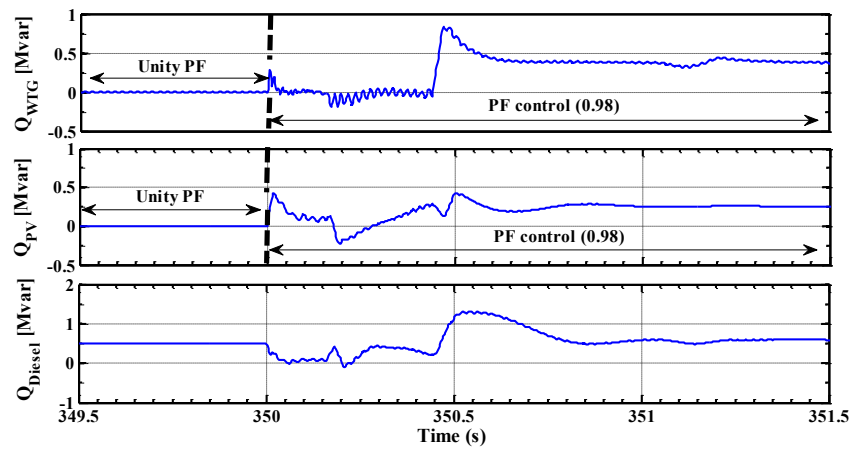

(a)

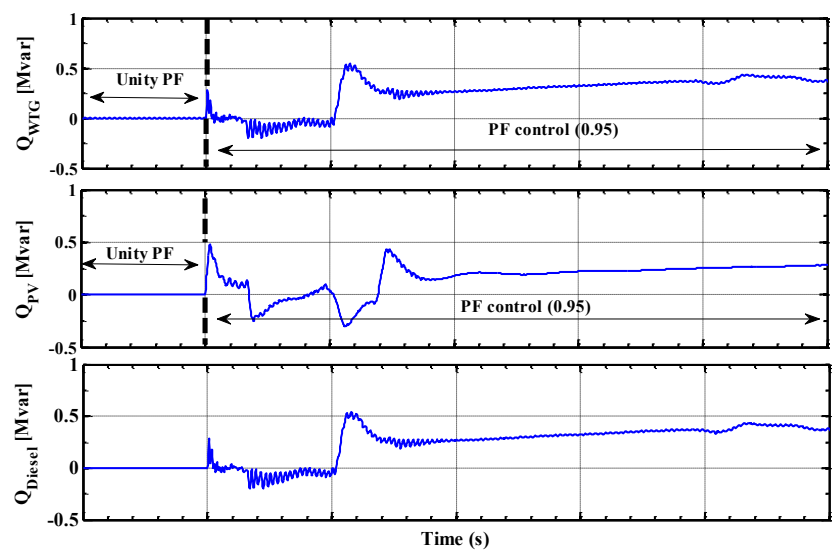

(b)

Fig.17. The reactive power compensation from DFIG-WT, PV and Diesel Generator at transition from unity to (a) $0.98 \mathrm{pf}$ (b) $0.95 \mathrm{pf}$.

Thus, the PV and DFIG-WT as shown in Fig. 17 (a,b) provided reactive power compensation to support the $\mathrm{MG}$ voltage whereas the CAP banks are at the maximum of 20 steps. Therefore, the reactive power compensation from the inverter-based DERs is needed to ensure better voltage profile. It can be concluded that the proposed CVC \& RPMS significantly enhancing the transient response and stability margin of the Hybrid MG during fault triggered islanding. It is worthy to mention that, while comparing the Cases $1 \& 2$ to the proposed Case 2 (CVC \& RPMS), the CAP banks moved lower and higher steps compared to Case 2 . That is, in Case 1 the CAP bank took less steps and in Case 3 it took higher steps than in Case 2. This emphasizes that not only the number of steps of slow devices decide the capability of the control rather its combination with voltage control of inverter-based DERs as full scheme.

\section{CONCLUSION}

In this paper, the German grid code in brief is introduced showing the requirements from DER units and MG at PCC with their dynamic support requirement and protection settings. The simulated cases $\mathrm{W} / \mathrm{O} \mathrm{CVC}$ and with $\mathrm{CVC}$ in the introduced test system are compared against each other in compliance with the German grid codes. The CVC scheme is introduced for a hybrid MG with diverse types of power generations, in addition to slow reactive power control devices without communication infrastructure. The CVC associated with RPMS demonstrates superior performance to regulate $\mathrm{MG}$ voltage by controlling the reactive power among DERs, OLTC, and Cap Banks for grid connected and islanded mode of operation. Also, the results showed satisfactory performance against the German grid codes, as the case with CVC complies within the voltage and frequency limits. The proposed CVC control achieves the same capability of the centralized controller by enhancing the voltage profile, maximizing fast dynamic reactive power reserve and transient response. Moreover, it improves the MCCT in response to a fault triggered islanding up to $230 \mathrm{~ms}$ and $360 \mathrm{~ms}$ during internal fault under islanded mode of operation. Further, the proposed CVC is compared against a thirds case with PF control. The proposed control showed superior performance regardless of the higher reactive power support from slow devices like CAP bank.

\begin{tabular}{|c|c|}
\hline \multicolumn{2}{|c|}{$\begin{array}{c}\text { VIII.APPENDIX } \\
\text { Table A.1 } \\
\text { PV model parameters }\end{array}$} \\
\hline Electron charge: & $q=1.6 \times 10^{-19} \mathrm{C}$ \\
\hline Shunt resistance: & $R_{s h}=1000 \Omega$ \\
\hline Ideality factor: & $A=1.5$ \\
\hline${ }^{\circledR}$ STC Short circuit current: & $I_{S C S}=2.5 \mathrm{~A}$ \\
\hline STC temperature: & $T_{C S}=298 \mathrm{~K}$ \\
\hline Boltzmann constant: & $k=1.38 \times 10^{-23} \mathrm{~J} / \mathrm{K}$ \\
\hline Series resistance: & $R_{s}=0.02 \Omega$ \\
\hline${ }^{8}$ STC Saturation current: & $I_{s}=1 \times 10^{-9} \mathrm{~A}$ \\
\hline Temperature coefficient: & $\alpha_{T}=0.001$ \\
\hline${ }^{\S}$ STC solar irradiance: & $E_{S T C}=1.0 \mathrm{~kW} / \mathrm{m}^{2}$ \\
\hline \multicolumn{2}{|c|}{ 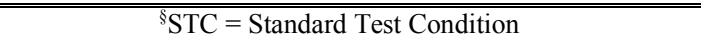 } \\
\hline \multicolumn{2}{|c|}{$\begin{array}{c}\text { Table A.2 } \\
\text { MPPT model parameters }\end{array}$} \\
\hline PV array short circuit current: & $I_{P V-S C}=160.1 \mathrm{~A}$ \\
\hline PV array open circuit voltage: & $V_{P V-O C}=750 \mathrm{~V}$ \\
\hline Sampling Interval: & $T_{\text {Sample }}=0.01 \mathrm{~s}$ \\
\hline Initial Value of $\mathrm{V}_{\mathrm{mpp}}$ : & $V_{\mathrm{mpp}}=1 \mathrm{kV}$ \\
\hline
\end{tabular}

\section{REFERENCES}

[1] P. Wang, D. H. Liang, J. Yi, P. F. Lyons, P. J. Davison and P. C. Taylor, "Integrating Electrical Energy Storage Into Coordinated Voltage Control Schemes for Distribution Networks," IEEE Trans. Smart Grid, vol.5, no.2, pp.1018-1032, March 2014.

[2] P. Mancarella, G. Chin Kim, and G. Strbac, "Evaluation of the impact of electric heat pumps and distributed CHP on LV networks," in Proc.2011 IEEE PowerTech, Trondheim, Norway, pp. 1-7.

[3] C. M. Hird, H. Leite, N. Jenkins and H. Li, "Network voltage controller for distributed generation," IEE Proceedings- Generation, Transmission and Distribution, vol.151, no.2, pp.150-156, March 2004.

[4] K. M. Son, K. S. Moon, S. K. Lee and J. K. Park "Coordination of an SVC with a ULTC reserving compensation margin for emergency control", IEEE Trans. Power Del., vol. 15, no. 4, pp.1193-1198, 2000.

[5] G. W. Kim and K. Y. Lee "Coordination control of ULTC transformer and STATCOM based on an artificial neural network", IEEE Trans. Power Syst., vol. 20, no. 2, pp.580 -586, 2005.

[6] J. Y. Park, S. R. Nam and J. K. Park, "Control of a ULTC Considering the Dispatch Schedule of Capacitors in a Distribution System," IEEE Trans. Power Syst., vol.22, no.2, pp.755-761, May 2007.

[7] M. E. Baran and I. M. El-Markabi, "A multiagent-based dispatching scheme for distributed generators for voltage support on distribution feeders", IEEE Trans. Power Syst., vol. 22, pp.52-59, 2007.

[8] M. Brenna, E. De Berardinis, L. Delli Carpini, F. Foiadelli, P. Paulon, P. Petroni, G. Sapienza, G. Scrosati and D. Zaninelli, "Automatic distributed voltage control algorithm in smart grids applications", IEEETrans. Smart Grid, vol. 4, pp.877-885, 2013.

[9] A. Kulmala, S. Repo and P. Jarventausta, "Increasing penetration of distributed generation in existing distribution networks using coordinated voltage control", Int. J. Distrib. Energy Resources, vol. 5, pp.227-255, 2009.

[10] P. H. Nguyen, J. M. A. Myrzik and W. L. Kling, "Coordination of voltage regulation in active networks", Proc. IEEE PES Transm. Distrib. Conf. Expo., 2008. 
[11] A. Kulmala, S. Repo and P. Järventausta, "Coordinated Voltage Control in Distribution Networks Including Several Distributed Energy Resources," IEEE Trans. Smart Grid, vol.5, no.4, pp. 2010-2020, July 2014.

[12] Kulmala, A.; Mutanen, A.; Koto, A.; Repo, S.; Jarventausta, P., "RTDS verification of a coordinated voltage control implementation for distribution networks with distributed generation," Innovative Smart Grid Technologies Conference Europe (ISGT Europe), 2010 IEEE PES , vol., no., pp.1,8, 11-13 Oct. 2010.

[13] A. Borghetti, M. Bosetti, S. Grillo, S. Massucco, C. A. Nucci, M. Paolone and F. Silvestro, "Short-term scheduling and control of active distribution systems with high penetration of renewable resources", IEEE Syst. $J$., vol. 4, pp.313-322, 2010.

[14] F. Pilo, G. Pisano and G. G. Soma, "Optimal coordination of energy resources with a two-stage online active management", IEEE Trans. Ind. Electron., vol. 58, pp.4526-4537, 2011.

[15] L. Yu, D. Czarkowski and F. de Leon, "Optimal distributed voltage regulation for secondary networks with DGs", IEEE Trans. Smart Grid, vol. 3, pp.959-967, 2012.

[16] A. Viehweider, H. Schichl, D. Burnier de Castro, S. Henein and D. Schwabeneder, "Smart robust voltage control for distribution networks using interval arithmetic and state machine concepts", Proc. Innov. Smart Grid Technol. Eur., 2010.

[17] A. Timbus, M. Larsson and C. Yuen, "Active management of distributed energy resources using standardized communications and modern information technologies", IEEE Trans. Ind. Electron., vol. 56, pp.4029-4037, 2009.

[18] R. Caldon, F. Rossetto and A. Scala, "Reactive power control in distribution networks with dispersed generators: A cost based method", Electr. Power Syst. Res., vol. 64, pp.209-217, 2003.

[19] A. A. Aquino-Lugo, R. Klump and T. J. Overbye, "A control framework for the smart grid for voltage support using agent-based technologies", IEEE Trans. Smart Grid, vol. 2, pp.173-180, 2011.

[20] D. Villacci, G. Bontempi and A. Vaccaro, "An adaptive local learning-based methodology for voltage regulation in distribution networks with dispersed generation", IEEE Trans. Power Syst., vol. 21, pp.1131-1140, 2006

[21] V. Galdi, A. Vaccaro and D. Villacci, "Voltage regulation in MV networks with dispersed generations by a neural-based multi objective methodology", Electr. Power Syst. Res., vol. 78, pp.785-793, 2008.

[22] T. Senjyu, Y. Miyazato, A. Yona, N. Urasaki and T. Funabashi, "Optimal distribution voltage control and coordination with distributed generation", IEEE Trans. Power Del., vol. 23, pp.1236-1242, 2008.

[23] N. Nimpitiwan and C. Chaiyabut, "Centralized control of system voltage/reactive power using genetic algorithm", Proc. Int. Conf. Intell. Syst. Appl. Power Syst., 2007.

[24] T. Niknam, A. M. Ranjbar and A. R. Shirani, "Impact of distributed generation on volt/var control in distribution networks", Proc. Power Tech, 2003.

[25] A. G. Madureira and J. A. Pecas Lopes, "Coordinated voltage support in distribution networks with distributed generation and microgrids", IET Renew. Power Gener., vol. 3, pp.439-454, 2009.

[26] A. Moawwad, V. Khadkikar and J. Kirtley, "A New P-Q-V Droop Control Method for an Inteline Photovoltaic (I-PV) Power system," IEEE Trans. Power Del., vol. 28, pp.658-668, 2013.

[27] EPRI, "EPRI Power Systems Dynamics Tutorial," Palo Alto, 2009.

[28] N. G. Hingorani and L. Gyugyi, in Understanding FACTS, M. E. El-Hawary, New York, IEEE, pp.135-205, 2000.

[29] A. Rabiee and M. Parniani, "Optimal reactive power dispatch using the concept of dynamic VAR source value," in Power \& Energy Society General Meeting, PES '09. IEEE, 2009.

[30] P. Kundur, Power System Stability and Control, McGraw-Hill,, 1994

[31] J. H. Choi, and S. L. Moon, "The Dead Band Control of LTC Transformer at Distribution Substation," IEEE Trans. Power Syst., vol. 24, pp.319-326, 2009.

[32] Manitoba HVDC Research Centre, "Single Phase Voltage Regulator", April $2007 . \quad$ [Online]. Available: https://hvdc.ca/uploads/ck/files/reference_material/Single_Phase_Regula tor.zip. [Accessed 18 May 2013].

[33] Bundesverband der Energie- und Wasserwirtschaft e.V. (BDEW), "Technische Richtlinie Erzeugungsanlagen am Mittelspannungsnetz Richtlinie fur Anschluss und Parallelbetrieb von Erzeugungsanlagen am Mittelspannungsnetz Ausgabe Juni 2008," BDEW, Berlin, 2008.

[34] M. Ropp, K. Aaker, J. Haigh and N. Sabbah, "Using power line carrier communications to prevent islanding [of PV power systems]," in Photovoltaic Specialists Conference, 2000. Conference Record of the Twenty-Eighth IEEE, 2000.

[35] R. Benato, R. Caldon and F. Censena, "Carrier signal-based protection to prevent dispersed generation islanding on MV systems," in CIRED, 2003.

[36] O. Abarrategui, I. Zamora, D. Larruskain and A. Iturregi, "Power line carrier communications and its interest in the current power grid scenario," $2008.2 \quad$ [Online]. Available: http://www.icrepq.com/icrepq-08/347-abarrategui.pdf. [Accessed 19 May 2013].

[37] N. Strath, "Islanding detection in power systems," 2005.

[38] M. Ropp, M. Begovic, A. Rohatgi, G. Kern, S. Bonn, R. H. and S. Gonzales, "Determining the relative effectiveness of islanding detection methods using phase criteria and non-detection zones," IEEE Trans. Energy Convers., vol. 15, no. 3, pp. 290-296, 2000.

[39] P. O'Kane and B. Fox, "Loss of mains detection for embedded generation by system impedance monitoring," in Developments in Power System Protection, Sixth International Conference, 1997.

[40] J. Vieira, W. Freitas, Z. Huand, W. Xu and A. Morelato, "Formulas for predicting the dynamic performance of ROCOF relays for embedded generation applications," IEE Proceedings Gen., Trans. and Dist., vol. 153, no. 4, pp.399-406, 2006.

[41] M. Redfern, O. Usta and G. Fielding, "Protection against loss of utility grid supply for a dispersed storage and generation unit," IEEE Trans. Power Del., vol. 8, no. 3, pp.948-954, 1993.

[42] F.S. Pai and S.J. Huang, "A detection algorithm for islanding-prevention of dispersed consumer-owned storage and generating units," IEEE Trans. Energy Conv., vol. 16, no. 4, pp.346-351, 2001.

[43] S. I. Jang and K. H. Kim, "An islanding detection method for distributed generations using voltage unbalance and total harmonic distortion of current," IEEE Trans. Power Del., vol. 19, pp.745-752, 2004.

[44] X. Zhu, G. Shen and D. Xu, "Evaluation of AFD islanding detection methods based on NDZs described in power mismatch space," in Energy Conversion Congress and Exposition, 2009. ECCE 2009. IEEE, 2009.

[45] H. Zeineldin and M. Salama, "Impact of Load Frequency Dependence on the NDZ and Performance of the SFS Islanding Detection Method," IEEE Trans. Ind. Elect., vol. 58, pp.139-146, 2011.

[46] J. Yin, L. Chang and C. Diduch, "A new adaptive logic phase-shift algorithm for anti-islanding protections in inverter-based DG systems," in Power Electronics Specialists Conference, 2005. PESC '05. IEEE 36th, 2005.

[47] L. A. C. Lopes and H. Sun, "Performance assessment of active frequency drifting islanding detection methods," IEEE Trans. Energy Conv., vol. 21, no. 1, pp.171-180, 2006.

[48] F. Liu, Y. Kang, Y. Zhang, S. Duan and X. Lin, "Improved SMS islanding detection method for grid-connected converters," Renewable Power Generation, IET, vol. 4, no. 1, pp. 36-42, 2010.

[49] S. K. Kim, J. H. Jeon, J. B. Ahn, B. Lee and S.H. Kwon, "Frequency-shift acceleration control for anti-islanding of a distributed-generation inverter," IEEE Trans. Ind. Elect., vol. 57, no. 2, pp. 494-504, 2010.

[50] S. K. Kim, J. H. Jeon, and H. K. Choi, "Design of dq-based voltage positive feedback for anti-islanding of a dg inverter," in Transmission \& Distribution Conference \& Exposition: Asia and Pacific, 2009, 2009. 